\title{
QUANTUM RECONSTRUCTION FOR FANO BUNDLES ON PROJECTIVE SPACE
}

\author{
ANDREW STRANGEWAY
}

\begin{abstract}
We present a reconstruction theorem for Fano vector bundles on projective space which recovers the small quantum cohomology for the projectivization of the bundle from a small number of low-degree Gromov-Witten invariants. We provide an extended example in which we calculate the quantum cohomology of a certain Fano 9-fold and deduce from this, using the quantum Lefschetz theorem, the quantum period sequence for a Fano 3-fold of Picard rank 2 and degree 24. This example is new, and is important for the Fanosearch program.
\end{abstract}

\section{$\S 1$. Introduction}

We say that a vector bundle $E$ is Fano if the total space of the projectivization, $X:=\mathbb{P}(E)$, is a Fano manifold. In this article we study rank $r$ Fano vector bundles on $n$-dimensional projective space $\mathbb{P}^{n}$. It is well known that the ring $H^{\bullet}(X ; \mathbb{Z})$ has two integer generating classes, $p$ and $\xi_{E}$, given by the tautological bundle on $\mathbb{P}^{n}$ and the relative tautological bundle on the projectivization $\mathbb{P}(E)$, respectively. These are canonical up to twisting of $E$ by $\mathcal{O}(d), d \in \mathbb{Z}$.

The Mori cone of $X$ has precisely two extremal rays $R_{1}, R_{2} \subset \overline{N E}_{X} \subset$ $H_{2}(X ; \mathbb{R})$. We make the assumption on the bundle $E$ (see Assumption 1 ) that the primitive generators of the rays $R_{1}, R_{2}$ form an integer basis of $H_{2}(X ; \mathbb{Z})$. We label this basis by $A_{1}, A_{2}$.

Without loss of generality, we may take $A_{2}$ to be the class of a line in the fiber. The representative for $A_{1}$ is less clear and indeed it is possible that it may not be represented by an algebraic curve (see [20, Question 3 and Example 4]). It is not clear whether for Fano bundles Assumption 1 always holds, although there exist counterexamples for Fano varieties in general.

Received February 23, 2013. Revised October 7, 2013. Accepted October 30, 2013.

First published online November 7, 2014.

2010 Mathematics Subject Classification. Primary 14N35, 53D45; Secondary 14J45.

(C) 2014 by The Editorial Board of the Nagoya Mathematical Journal 
It follows from Assumption 1 that there exists $d \in \mathbb{Z}$ such that for $X:=$ $\mathbb{P}(E \otimes \mathcal{O}(d))$, the basis $A_{1}, A_{2}$ of $H_{2}(X ; \mathbb{Z})$ is dual to the basis $p, \xi_{E \otimes \mathcal{O}(d)}$ of $H^{2}(X ; \mathbb{Z})$. In the following we will assume that $E$ is normalized in this manner, and we will denote the tautological class by $\xi$ without reference to the corresponding bundle.

Given Assumption 1, we determine the Gromov-Witten invariants with target class represented by curves in the fiber of the bundle map. This generalizes some early results of Qin and Ruan [25]. We then impose an additional assumption on our normalized bundle (see Assumption 2): that $r+1+c_{1}>$ 0 , where $c_{1}(E)=c_{1} p$. This assumption is essentially an assumption on the rank of the bundle, and holds automatically in the case $r>n$. Assumption 2 ensures that quantum multiplication of two classes $\alpha, \beta \in H^{\bullet}(X ; \mathbb{Z})$ with $\operatorname{deg}(\alpha)+\operatorname{deg}(\beta) \leq n$ has no quantum corrections determined by GromovWitten invariants with class $a A_{1}+b A_{2}$, with $a$ and $b$ both nonzero.

We show (see Theorem 1 ) that for a Fano bundle $E$ satisfying Assumptions 1 and 2, the small quantum cohomology of $X$ is determined by the Gromov-Witten invariants with class $A \in H_{2}(X)$ of degree $-K \cdot A \leq n+1$. This gives us good control over the invariants required to determine the quantum cohomology. To place such a condition seems reasonable, as evidence suggests that low-rank Fano bundles are split (see [2]). The projectivization of a split bundle on $\mathbb{P}^{n}$ is toric, so the quantum cohomology is determined by an existing theory of Givental [14, Theorem 0.1].

In the second part of this article, we provide an extended example, using Theorem 1 to calculate the quantum cohomology for the Fano 9-fold $\mathbb{P}\left(\bigwedge^{2} \Omega_{\mathbb{P}^{4}}\right)$, the projectivization of second wedge of the cotangent bundle on $\mathbb{P}^{4}$. From the quantum multiplication data we produce the small $J$-function, following Guest in [17] and [18]. We observe in the $J$-function some tantalizing traces of modularity, in the form of the Apéry numbers.

We apply the quantum Lefschetz theorem (see [10, Theorem 2, Corollary 6]) to the result for the 9 -fold to compute the quantum period for the rank 2 Fano 3-fold No. 17 in the Mori-Mukai list (see [23, Table 2]), which can be given as a complete intersection in the total space of the projectivized bundle. This result is important in the classification of Fano 3-folds as carried out in the Fanosearch program (see [8]).

While preparing the first draft of this paper, the method we describe here was the only way to obtain the quantum cohomology of this Fano 3-fold. More recently, Coates, Corti, Galkin, Golyshev, and Kasprzyk have shown that the quantum period of this Fano 3-fold can be computed using 
abelian/nonabelian correspondence (see [6]). Nonetheless the method presented here is less taxing than the alternative approach, and the methods presented in this article also apply to more general Fano bundles.

\section{$\S 2 . \quad$ Notation and assumptions}

Throughout this article we will use Fulton's convention for projectivized bundles; we regard $\mathbb{P}(E)$ as the bundle of 1-dimensional linear subspaces in the fibers of $E$, not 1-dimensional quotients. Since we work exclusively with cohomology in even degree, we say that $\alpha$ has degree $n / 2$ if $\alpha \in H^{n}$.

\subsection{Fano bundles and extremal rays}

A vector bundle $E$ is defined to be Fano if its projectivization, $\mathbb{P}(E)$, is a Fano manifold. Let $E \rightarrow \mathbb{P}^{n}$ be a Fano bundle of rank $r$. Let $p=c_{1}\left(\mathcal{O}_{\mathbb{P} n}(1)\right)$ be the hyperplane class on $\mathbb{P}^{n}$, and let $\xi_{E}=c_{1}\left(\mathcal{O}_{\mathbb{P}(E)}(1)\right)$ be the relative hyperplane class on $\mathbb{P}(E)$. We will write $c_{i}(E)=c_{i} p^{i}$ for the Chern classes of $E$.

Let $X=\mathbb{P}(E)$, and let $P: X \rightarrow \mathbb{P}^{n}$ be the projection map. We have that the anticanonical class is given by

$$
-K_{X}=(n+1) p+c_{1}(E)+r \xi_{E}
$$

Note that while it might appear that $-K_{X}$ depends on the normalization of $E$, one can easily check that for $E^{\prime}=E \otimes \mathcal{O}(d), c_{1}\left(E^{\prime}\right)=c_{1}(E)+r d p$, while $\xi_{E}^{\prime}=\xi_{E}-d p$

We fix a basis for $H^{\bullet}(X ; \mathbb{Z}), \phi_{1}, \ldots, \phi_{r(n+1)}$, generated as a ring by $\mathbf{1}, p$, and $\xi_{E}$. This basis is given lexicographic ordering where we take $p$ before $\xi_{E}$. Let $(\cdot, \cdot)$ denote the intersection pairing on cohomology, so that $(\alpha, \beta)=$ $\int_{M} \alpha \cup \beta$. We denote $\phi^{i}$ the basis dual to $\phi_{i}$ defined by this paring. We will regularly denote the cup product of cohomology classes implicitly.

As $X$ is the projectivization of a vector bundle over projective space, the Mori cone of $X, \overline{N E}_{X}$, has exactly two extremal rays $R_{1}, R_{2}$ (see [7, p. 25]). We may take primitive generators of the rays $A_{1}, A_{2} \in H_{2}(X ; \mathbb{Z})$ such that $R_{1}=\mathbb{R}_{+} A_{1}$ and $R_{2}=\mathbb{R}_{+} A_{2}$. Up to a choice of ordering, $A_{2}$ is represented by a line in the fiber of $P: A_{2}=\operatorname{PD}\left(p^{n} \xi^{r-2}\right)$. The form that $A_{1}$ takes in general is less clear and, as we noted in the Introduction, it may not be representable as an algebraic curve. We will make the following assumption on the generators of the extremal rays of $X$. 
Assumption 1. We assume the following equivalent conditions:

- $A_{1}, A_{2} \in H_{2}(X ; \mathbb{Z})$ form an integral basis,

- $p \cdot A_{1}=1$.

Assumption 1 implies that $A_{1}=\mathrm{PD}\left[p^{n-1} \xi_{E}^{r-1}+\left(k+c_{1}\right) p^{n} \xi_{E}^{r-2}\right]$ for some integer $k$. A sufficient condition for Assumption 1 to hold is if the class $A_{1}$ is realized by rational curves corresponding to lines in the base $\mathbb{P}^{n}$. This occurs when $A_{1}$ is the class of a section of $\left.P\right|_{\ell}$ for $\ell \subset \mathbb{P}^{n}$ a line. Note that $\ell$ need not be a generic line: unless the bundle is uniform it is reasonable to expect that any line realizing $A_{1}$ will be some jumping line for the vector bundle $E$ (see [1]). In greater generality one might expect that $A_{1}$ would be the class of a section of $\left.P\right|_{C}$, for some curve $C$ of degree greater than 1 , that is, corresponding to a jumping conic or higher-degree rational curve. This would contravene Assumption 1. At present we do not have examples of such a situation, although we have no general statement to rule it out.

Given Assumption 1, it trivially follows that

$$
\begin{aligned}
p \cdot A_{1} & =1, \quad p \cdot A_{2}=0, \\
\xi_{E} \cdot A_{1} & =k, \quad \xi_{E} \cdot A_{2}=1 .
\end{aligned}
$$

By choosing the basis $p,(\xi-k p)$, this product becomes diagonal. This is equivalent to twisting $E$ by $\mathcal{O}(k)$, which does not change $\mathbb{P}(E)$. For the rest of this article, we assume that $E$ is normalized in this way so that the product is diagonalized, that is,

$$
\begin{aligned}
p \cdot A_{1} & =1, \quad p \cdot A_{2}=0, \\
\xi_{E} \cdot A_{1} & =0, \quad \xi_{E} \cdot A_{2}=1 .
\end{aligned}
$$

Under these conditions we are therefore free to assume that $E$ is normalized such that $A_{1}=\operatorname{PD}\left[p^{n-1} \xi_{E}^{r-1}+c_{1} p^{n} \xi_{E}^{r-2}\right]$ and $A_{2}=\operatorname{PD}\left(p^{n} \xi^{r-2}\right)$, and we do so in what follows, dropping the subscript $E$ on $\xi$. Note that this normalization implies that $\xi$ is nef while $\xi-p$ is not.

Since $E$ is a Fano bundle, $-K_{X}=\left(n+1+c_{1}\right) p+r \xi$ is ample. We therefore have

$$
-K_{x} \cdot A_{1}=n+1+c_{1}>0 .
$$

With this normalization fixed, we will later also make the following assumption.

Assumption 2. It holds that $E$ satisfies $r+1+c_{1}>0$. 
This assumption is essentially a condition on the rank of the bundle and is fulfilled if $r>n$ (combining this with (1), Assumption 2 follows easily). The purpose of this assumption will be made more transparent in the following section, as it pertains to the degree of certain quantum corrections.

We will denote by $P$ and $\Xi$ the extremal contractions given by the linear systems induced by $p$ and $\xi$, respectively:

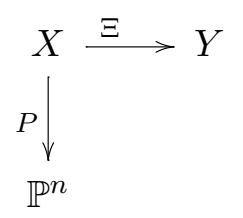

\subsection{Gromov-Witten and quantum cohomology}

We briefly review some aspects of Gromov-Witten theory to fix notation. In the following, we use the fact that $A_{1}, A_{2}$ form an integer basis for $H_{2}(X ; \mathbb{Z})$.

Let $X_{0, n, a A_{1}+b A_{2}}$ denote the moduli space of $n$-pointed genus 0 stable maps to $X$ of image class $a A_{1}+b A_{2}$ from curves of genus 0 with $n$ marked points (see [21], [12]). There are evaluation maps ev $i: X_{0, n, a A_{1}+b A_{2}} \rightarrow X, 1 \leq$ $i \leq n$ and, given cohomology classes $\alpha_{1}, \alpha_{n} \in H^{\bullet}(X)$, we define genus 0 Gromov-Witten invariants as

$$
\left\langle\alpha_{1}, \ldots, \alpha_{n}\right\rangle_{0, n, a A_{1}+b A_{2}}^{X}:=\int_{\left[X_{0, n, a A_{1}+b A_{2}}\right]^{\mathrm{vir}}} \mathrm{ev}_{1}^{*} \alpha_{1} \wedge \cdots \wedge \mathrm{ev}_{n}^{*} \alpha_{n}
$$

where $\left[X_{0, n, a A_{1}+b A_{2}}\right]^{\mathrm{vir}}$ is the virtual fundamental class of $X_{0, n, a A_{1}+b A_{2}}$ (see [22], [4], [3]). Recall that the virtual dimension of $X_{0, n, a A_{1}+b A_{2}}$ is

$$
\operatorname{dim}_{\mathrm{vir}}\left(X_{0, n, a A_{1}+b A_{2}}\right)=\operatorname{dim}(X)-3+n-K \cdot\left(a A_{1}+b A_{2}\right) .
$$

The small quantum product on a manifold $M$ is a deformation of the usual (classical) product in cohomology. The deformation takes the form of quantum corrections, which are governed by genus 0 Gromov-Witten invariants.

Let $\Lambda:=\mathbb{C}\left[H^{2}(X ; \mathbb{Z})\right]$, an element of which is a finite sum $\sum_{d \in H^{2}(X ; \mathbb{Z})} \lambda_{d} q^{d}$, with symbols multiplying as follows: $q^{d_{1}} q^{d_{2}}=q^{d_{1}+d_{2}}$. We let $q_{1}=q^{A_{1}}$ and $q_{2}=q^{A_{2}}$ be the elements of $\Lambda$ associated to $A_{1}$ and $A_{2}$ corrections, respectively.

We consider the small quantum product as the following operation:

$$
\star: H^{\bullet}(X ; \mathbb{C}) \times H^{\bullet}(X ; \mathbb{C}) \rightarrow H^{\bullet}(X ; \mathbb{C}) \otimes \Lambda
$$


with

$$
\alpha \star \beta=\sum_{i=1}^{(n+1) r} \sum_{a, b \geq 0}\left\langle\alpha, \beta, \phi_{i}\right\rangle_{0,3, a A_{1}+b A_{2}}^{X} \phi^{i} q_{1}^{a} q_{2}^{b} .
$$

We define $d_{1}=\operatorname{deg}\left(q_{1}\right)=-K \cdot A_{1}=n+1+c_{1}$ and $d_{2}=\operatorname{deg}\left(q_{2}\right)=-K$. $A_{2}=r$. With this definition, the quantum product makes $H^{\bullet}(X, \Lambda)$ into a graded ring. This fact, which is a simple consequence of the virtual dimension of $X_{0, n, a A_{1}+b A_{2}}$, constrains the degree of image class for the GromovWitten invariants associated to quantum corrections based on the degree of the classes being multiplied. We will often refer to the degree-preserving nature of quantum multiplication by little more than for degree reasons. To calculate small quantum multiplication by $p$ and $\xi$, we need only consider two-point invariants, due to the divisor axiom (see [3]).

Note that Assumption 2 implies that $d_{1}+d_{2}>n$, so the quantum multiplication of two classes $\alpha, \beta \in H^{\bullet}(X ; \mathbb{Z})$ with $\operatorname{deg}(\alpha)+\operatorname{deg}(\beta) \leq n$ has no quantum corrections determined by Gromov-Witten invariants with class $a A_{1}+b A_{2}$, with $a$ and $b$ both nonzero.

\section{§3. Reconstruction theorem for Fano bundles}

Lemma 1. Let $X=\mathbb{P}(E)$ for $E \rightarrow Y$, a Fano bundle (not necessarily on $\left.\mathbb{P}^{n}\right)$ of rank $r$, and let $Y$ be smooth of dimension $n$. Let $\xi$ be the relative hyperplane class on $X$, let $\pi: X \rightarrow Y$ be the induced bundle map, and let $A=\mathrm{PD}\left(p^{n} \xi^{r-2}\right)$ be the extremal curve corresponding to lines in the fiber of $\pi$. The Gromov-Witten invariants of type $\langle\alpha, \beta\rangle_{0,2, k A}^{X}$ vanish for $k \geq 2$. Furthermore, $\langle\alpha, \beta\rangle_{0,2, A}^{X}=\pi_{*} \alpha \cdot \pi_{*} \beta$.

Proof. This result generalizes [25, Lemmas 3.6 and 3.7].

We demonstrate that, for $k \geq 2$, there do not exist any rational curves of class $k A$ which satisfy the intersection properties given by $\alpha$ and $\beta$. Connected curves of the class $k A$ are restricted to live in a single fiber of $\pi$. Since we require that the curve intersects $\operatorname{PD}(\alpha)$ and $\operatorname{PD}(\beta)$, such a curve may only exist if $\operatorname{PD}(\alpha)$ and $\operatorname{PD}(\beta)$ both intersect a common fiber of $\pi$. We provide a dimension-counting argument to show that no such common fiber exists.

The virtual dimension of $X_{0,2, k A}$ is

$$
\operatorname{dim}_{\mathrm{vir}}\left(X_{0,2, k A}\right)=\operatorname{dim}(X)-3+2+k \operatorname{deg}\left(q^{A}\right)=n+r-2+k r .
$$

If $k \geq 2$, then, for degree reasons, we only get nonzero invariants if $\operatorname{deg}(\alpha)+$ $\operatorname{deg}(\beta)=n+r-2+k r \geq n+r-2+2 r$. 
Letting $s$ and $t$ be the dimensions of $\operatorname{PD}(\alpha)$ and $\operatorname{PD}(\beta)$, respectively, we have $s+t \leq n+r-2 r<n$. This implies that $\mathrm{PD}(\alpha)$ and $\mathrm{PD}(\beta)$ do not intersect in a common fiber of $\pi$; in particular, $\pi_{*} \operatorname{PD}(\alpha)$ and $\pi_{*} \operatorname{PD}(\beta)$ do not intersect generically in $Y$. Since there are no rational curves with the correct intersection properties, the associated Gromov-Witten invariants vanish.

The Gromov-Witten invariants $\langle\alpha, \beta\rangle_{0,2, A}^{X}$ count genuine lines in the fibers of $\pi$ which intersect sufficiently generic cycles representing the classes $\operatorname{PD}(\alpha)$ and $\operatorname{PD}(\beta)$. The fibers are projective space, and it is well known that the only nonzero, two-point invariants for projective space encode the fact that there is exactly one line between two points (see, e.g., [19]). So we can rephrase the issue here: we wish to count fibers of $P$ which contain a point of the cycle representing $\operatorname{PD}(\alpha)$ and a point of the cycle representing $\operatorname{PD}(\beta)$. This can be calculated via the ordinary intersection product after pushdown by $P$, as stated.

Note that if $\alpha, \beta$ are elements of the basis $\left\{\phi_{i}\right\}$,

$$
\langle\alpha, \beta\rangle_{0,2, A_{2}}= \begin{cases}1 & \text { if } \alpha=p^{n-l} \xi^{r-1}, \beta=p^{n} \xi^{r-1}, 1 \leq l \leq n \\ 0 & \text { otherwise. }\end{cases}
$$

We prove two reconstruction lemmas, which together allow us to produce the full quantum cohomology for $X$ from a small number of input GromovWitten invariants. Note that throughout we are heavily reliant upon the assumption that the product between divisor classes and extremal curve classes is diagonal. The $\xi$-lemma tells us that if we have the quantum multiplication of a class by $p$, then we know for free the multiplication of the same class by $\xi$. The $p$-lemma says that if we know $\xi \star p^{k} \xi^{l}$, then we get $p \star p^{k-1} \xi^{l+1}$. Note that once we have determined quantum multiplication by divisor classes, we have determined the entirety of quantum cohomology, so we restrict our interest to quantum multiplication by divisors. Since quantum multiplication is distributive, we need consider only multiplication of basis elements.

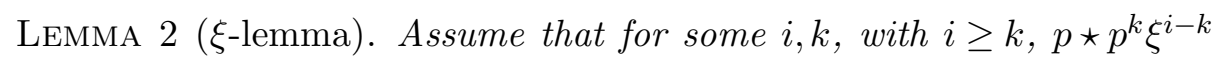
is known. We can then calculate $\xi \star p^{k} \xi^{i-k}$ with no geometric (i.e., moduli space) calculation. Put differently, we already have sufficient GromovWitten invariants to calculate the quantum corrections in $\xi \star p^{k} \xi^{i-k}$. 
Proof. For degree reasons, the quantum corrections in $p \star p^{k} \xi^{d-k}$ are determined by (and hence determine) all Gromov-Witten invariants of the form

$$
\langle\alpha, \beta\rangle_{0,2, a A_{1}+b A_{2}}^{X},
$$

with $a d_{1}+b d_{2} \leq i+1$ and $a \geq 1$.

Likewise, the quantum corrections for $\xi \star p^{k} \xi^{i-k}$ are determined by invariants of the form

$$
\langle\alpha, \beta\rangle_{0,2, a A_{1}+b A_{2}}^{X},
$$

with $a d_{1}+b d_{2} \leq i+1$ and $b \geq 1$.

It is clear that the only Gromov-Witten invariants required to determine the quantum corrections in $\xi \star p^{k} \xi^{i-k}$, which are not already determined by $p \star p^{k} \xi^{i-k}$, are $\langle\alpha, \beta\rangle_{0,2, A_{2}}^{X}$, which are given by Lemma 1 .

Lemma 3 (p-lemma). Assume that all quantum multiplication by divisor classes of classes of degree at most $i-1$ is known. Further, assume that $\xi \star p^{k+1} \xi^{i-k-1}, i-k-1 \geq 0$, is known. Then we can calculate $p \star p^{k} \xi^{i-k}$ with no geometric calculation. Note that for $i \geq n+1$ and $k=n, \xi \star p^{n+1} \xi^{i-n-1}=$ 0 , since $p^{n+1}=0$.

Proof. Since quantum cohomology is both associative and commutative, we can use the knowledge of multiplication in lower degree to make the following manipulations:

$$
\begin{aligned}
p \star p^{k} \xi^{i-k} & =p \star\left(\xi \star p^{k} \xi^{i-k-1}-f_{2} q_{2}\right) \\
& =\xi \star p^{k+1} \xi^{i-k-1}+\xi \star f_{1} q_{1}-p \star f_{2} q_{2} \\
& =p^{k+1} \xi^{i-k}+\text { known quantum corrections, }
\end{aligned}
$$

where $f_{1}$ is the quantum correction from $p \star p^{k} \xi^{i-k-1}$ and $f_{2}$ the correction from $\xi \star p^{k} \xi^{i-k-1}$. Note that these are of degree $i-d_{1}$ and $i-d_{2}$, respectively, so their multiplication by divisors is, by assumption, known. At each step the quantum corrections are all known and governed by invariants we already have, since they are necessarily of lower degree.

For degree reasons, quantum multiplication, by divisor classes, of classes of degree $i \leq n$ is completely determined by the Gromov-Witten invariants of the form $\langle\alpha, \beta\rangle_{0,2, A_{2}}^{X}$ and $\langle\alpha, \beta\rangle_{0,2, k A_{1}}^{X}$ for $k d_{1} \leq n+1$. Note in particular that there can be no corrections coming from $\langle\alpha, \beta\rangle_{0,2, A_{1}+A_{2}}^{X}$ invariants. 
Every basis element of degree $i \geq n+1$ is divisible by $\xi$, so we can apply the $p$-lemma, the proof of which requires that we divide out a factor of $\xi$.

In the process of producing an algorithm from Lemmas 2 and 3 to carry out the reconstruction process, we consider cohomology classes as vectors in the lexicographical basis $\phi_{i}$. Quantum multiplication by the basis classes $p$ and $\xi$ can be considered as left multiplication of cohomology vectors by $r(n+1) \times r(n+1)$ matrices $M_{p}$ and $M_{\xi}$, respectively.

With this viewpoint in mind, we see that the preceding lemmas can be reinterpreted.

LEMMA 4 ( $p$-lemma as linear algebra). Given the ith column of $M_{p}$, then the ith column of $M_{\xi}$ may be determined by linear algebra.

LEMma 5 ( $\xi$-lemma as linear algebra). Assume that the first $i$ columns of $M_{p}$ and $M_{q}$ have been determined; then the $(i+1)$ column of $M_{p}$ can be determined using linear algebra.

Theorem 1. Let $X=\mathbb{P}(E)$ with $E \rightarrow \mathbb{P}^{n}$ be a Fano bundle of rank $r$, such that $r+c_{1}(E)>0$. The quantum cohomology of $X$ can be reconstructed from two sets of Gromov-Witten invariants: those already determined by Lemma 1 and those of the form $\left\langle p^{i}, \alpha\right\rangle_{0,2, k A_{1}}^{X}$ for $0 \leq i \leq n, \alpha \in H^{\bullet}(X ; \mathbb{Z})$ and $k d_{1} \leq n+1$. Phrased differently, the quantum multiplication can be reconstructed from the multiplication $p \star p^{i}$ for $0<i \leq n$.

Proof. The proof proceeds by the construction of an algorithm. We alternately apply Lemma 3 followed by Lemma 2 to calculate the multiplication for all basis elements following the lex ordering. We produce this algorithm in pseudocode below. Note that the invariants stated in the hypothesis are precisely those which define the multiplication $p \star p^{i}$ for $0 \leq i \leq n$.

We first describe in words roughly how the algorithm proceeds. Assume that the multiplication of classes of degree at most $i-1$ is determined. Consider the first basis element of degree $i$ (under lexicographical ordering). The class is then necessarily of the form $p^{i}$ if $i \leq n$ or $p^{n} \xi^{i-n}$ if $i>n$. In the first case we are in the special case of Lemma $3\left(p^{n+1}=0\right)$ and we obtain $p \star p^{n} \xi^{i-n}$. In the second case we have $p \star p^{i}$ by hypothesis.

We are now in a position to apply Lemma 2, to obtain the $\xi$ multiplication of this class. One can easily check that we can now apply both lemmas to the next basis element in degree $i$ and so on. After obtaining the multiplication data for classes of degree $i$, we repeat the process for degree $i+1$ (again, either obtaining the $p$ multiplication as a special case of the $p$-lemma or 
knowing it by hypothesis). We repeat until we obtain the full quantum multiplication data.

The input for the reconstruction process is a pair of $r(n+1) \times r(n+1)$ matrices $M_{p}$ and $M_{\xi}$. In $M_{p}$, the columns corresponding to multiplication $p \star p^{i}$ for $0 \leq i \leq n$ are known, and we initialize the unknown entries as zero. We can initialize $M_{\xi}$ as the zero matrix. By convention, we label arrays with the first entry given index 1. We may think of the reconstruction process as giving an algorithm to fill in the rest of the matrices $M_{p}$ and $M_{\xi}$.

The result of Lemma 3 can usefully be written as

$$
p \star p^{k} \xi^{i-k}=\xi \star p \star p^{k} \xi^{i-k-1}-p \star\left(\xi \star p^{k} \xi^{i-k-1}-p^{k} \xi^{i-k}\right) .
$$

We use this form in the algorithm as it lends itself easily to calculation by matrix multiplication.

We define the following functions for use in our pseudocode. Let numelts $(d)$ be the number of basis elements $\phi_{i}$ in degrees less than or equal to $d$ :

$$
\text { numelts }(d)= \begin{cases}\frac{1}{2}(d+1)(d+2) & \text { for } d \leq n \text { and } d \leq r-1 \\ d+1+n\left(d+\frac{n}{2}+\frac{1}{2}\right) & \text { for } d>n \text { and } d \leq r-1 \\ d+1+(r-1)\left(d-\frac{r}{2}+1\right) & \text { for } d \leq n \text { and } d>r-1 \\ (d+1)\left(1-\frac{d}{2}\right)+n\left(d-\frac{n}{2}+\frac{1}{2}\right) & \\ +(r-1)\left(d-\frac{r}{2}+1\right) & \text { for } d>n \text { and } d>r-1\end{cases}
$$

Let $\mathrm{I}(a, b)$ be the position that $p^{a} \xi^{b}$ appears in the basis of $H^{\bullet}(X)$, when given lexicographical ordering. This is given by

$$
\mathrm{I}(a, b)= \begin{cases}\operatorname{numelts}(a+b-1)+(b+1) & \text { for } a+b \leq n \\ \operatorname{numelts}(a+b-1)+(n-1+a) & \text { for } a+b>n\end{cases}
$$

Finally we define the term function, which extracts from a polynomial the term specified along with the corresponding coefficient. It is given by the expression Term(polynomial, monomial) (e.g., Term $\left(5 x^{2}+3 x y+2 x+1\right.$, $x)=2 x)$.

Let $C$ be an $r$-vector with $C[i]:=-c_{i}(E)$; we use the entries to $-c_{i}(E)$ to determine the form $\xi^{r}$ takes.

We produce the following algorithm. 
\#\# d loops over all the degrees to calculate

for $d=0 \rightarrow r+n$ do

\#\# We loop over all classes in degree $\mathrm{d}$

\#\# using the numelts function to determine the number of classes in degree $\mathrm{d}$

for $k=0 \rightarrow($ numelts $(d)-\operatorname{numelts}(d-1)-1)$ do

\#\# amax $:=\bar{a}$ and bmin $:=\underline{b}$ determine the exponent of elements in the degree.

$\bar{a}:=\min (d, n)$

$\underline{b}:=\max (d-n, 0)$

\#\# test to see if $p$-lemma should be applied

\#\# to rule out columns corresponding to multiplication of $p^{d}$

if $d>n$ or $k>0$ then

$\mathrm{HOLD}:=M_{\xi} M_{p} V_{(\bar{a}-k, \underline{b}+k-1)}-M_{p}\left(M_{\xi} V_{(\bar{a}-k, \underline{b}+k-1)}-V_{(\bar{a}-k, \underline{b}+k)}\right)$ \#\# the following loop copies HOLD into the appropriate column of $M_{p}$

$$
\begin{aligned}
& \text { for } j=1 \rightarrow(n+1) * r \text { do } \\
& \quad P[j, \mathrm{I}(\bar{a}-k, \underline{b}+k)]:=\operatorname{HOLD}[j]
\end{aligned}
$$

end for

\section{end if}

\#\# Applying $\xi$-lemma

\#\# (can improve on the bound $s$ )

for $s=1 \rightarrow(n+1) r$ do

\#\# Extracting the quantum corrections from the relevant

\#\# $p$ multiplication. We just pull out the data

\#\# from the corresponding column in $M_{p}$

\#\# Note the important factor $\frac{1}{s}$

\#\# from the divisor axiom

for $j=1 \rightarrow(n+1) r$ do

$$
\begin{aligned}
M_{\xi}[j, \mathrm{I}(\bar{a}-k, \underline{b}+k)]:= & M_{\xi}[j, \mathrm{I}(\bar{a}-k, \underline{b}+k)] \\
& +\frac{1}{s} \operatorname{Term}\left(M_{p}[j, \mathrm{I}(\bar{a}-k, \underline{b}+k)], s\right)
\end{aligned}
$$

end for

end for 
\#\# Testing for special case where class $=p^{\alpha} \xi^{r-1}$

if $\underline{b}+k=r-1$ then

\#\# Insert additional quantum correction in special case

$M_{\xi}[\mathrm{I}(\bar{a}-k, 0), \mathrm{I}(\bar{a}-k, \underline{b}+k)]:=q_{2}$

\#\# Insert classical multiplication in special case

\#\# i.e. copy across some Chern classes

\#\# Note that we shift the entries as we copy them to account \#\# for the powers of $p$ that our class includes

\#\# $s$ is restricted so that we account for the vanishing of $p^{n+1}$

$$
\begin{aligned}
& \text { for } s=1 \rightarrow n+r-1-d \text { do } \\
& \qquad M_{\xi}[\mathrm{I}(\bar{a}-k+s, r-s), \mathrm{I}(\bar{a}-k, \underline{b}+k)]:=C[s]
\end{aligned}
$$

end for

else

\#\# Insert classical multiplication in generic case

$M_{\xi}[\mathrm{I}(\bar{a}-k, \underline{b}+k+1), \mathrm{I}(\bar{a}-k, \underline{b}+k)]:=1$

end if

end for

end for

\subsection{Special cases}

There are two special cases in which Theorem 1 can be significantly strengthened. The first is when the second extremal contraction realizes $X$ as the projectivization of a vector bundle on some other space, the second when the contraction realizes $X$ as the blowup of some smooth space in a smooth locus. In both cases, the entire quantum cohomology is determined by counting lines in the fibers of the two extremal maps $P$ and $\Xi$. We first prove a lemma regarding the Gromov-Witten invariants of a blowup of a smooth subvariety in a smooth ambient space.

Lemma 6. Let $Y$ be a smooth algebraic variety of dimension $n$, let $Z \subset Y$ be a smooth, connected subvariety of codimension $r \geq 2$, and let $X:=\mathrm{Bl}_{Z} Y$ be the blowup of $Y$ along $Z$. Let $A_{1}$ be the extremal ray contracted by the blow-up map $\Xi: X \rightarrow Y$. We have that $A_{1}$ is the class of a line in the fiber of the exceptional divisor $D$ of the blowup. Then, for any $\alpha_{1}, \alpha_{2} \in H^{\bullet}(X ; \mathbb{Z})$, 
with $\operatorname{deg}\left(\alpha_{1}\right)+\operatorname{deg}\left(\alpha_{2}\right)=\operatorname{dim}_{\mathrm{vir}}\left(X_{0,2, k A_{1}}\right)$,

$$
\left\langle\alpha_{1}, \alpha_{2}\right\rangle_{0,2, k A_{1}}^{X}= \begin{cases}\left.\left.\Xi\right|_{*} \iota^{*} \alpha_{1} \cdot \Xi\right|_{*} \iota^{*} \alpha_{2} & k=1, \\ 0 & k \geq 2,\end{cases}
$$

where $\Xi \mid$ is the restriction of $\Xi$ to $D \subset X$, the exceptional divisor of the blowup, and $\iota: D \hookrightarrow X$ is the embedding of $D$ in $X$.

Proof. We summarize the geometry of the situation in the following diagram:

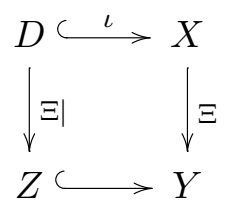

Note that $D=\mathbb{P}\left(N_{Z / Y}\right)$ is the projectivization of the normal bundle to $Z$ in $Y$, so we are in a similar situation to that discussed in Lemma 1.

We first prove vanishing for $k \geq 2$ using a push-pull argument.

Consider the following diagram of morphisms:

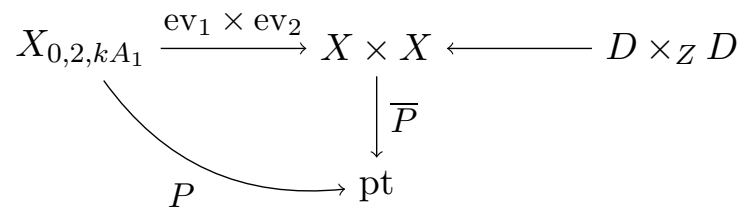

The Gromov-Witten invariant $\left\langle\alpha_{1}, \alpha_{2}\right\rangle_{0,2, k A_{1}}$ is given by

$$
P_{*}\left(\left[X_{0,2, k A_{1}}\right]^{\mathrm{vir}} \cap\left(\mathrm{ev}_{1} \times \mathrm{ev}_{2}\right)^{*}\left(\alpha_{1} \otimes \alpha_{2}\right)\right) .
$$

Since $P$ is the map to a point, the above is an integral as expected. If we can show that the integrand vanishes, we have shown vanishing for the Gromov-Witten invariant. We factor $P$ through the above diagram as $\bar{P} \circ\left(\mathrm{ev}_{1} \times \mathrm{ev}_{2}\right)$, since the map to a point is unique. We manipulate this expression using the projection formula (see [11, p. 34]):

$$
\begin{aligned}
& P_{*}\left(\left[X_{0,2, k A_{1}}\right]^{\mathrm{vir}} \cap\left(\mathrm{ev}_{1} \times \mathrm{ev}_{2}\right)^{*}\left(\alpha_{1} \otimes \alpha_{2}\right)\right) \\
& \quad=\bar{P}_{*}\left(\left(\mathrm{ev}_{1} \times \mathrm{ev}_{2}\right)_{*}\left[X_{0,2, k A_{1}}\right]^{\mathrm{vir}} \cap\left(\alpha_{1} \otimes \alpha_{2}\right)\right) .
\end{aligned}
$$

Since connected curves of class $k A_{1}$ are restricted to a single fiber of $\Xi \mid$, it is clear that the image $\left(\mathrm{ev}_{1} \times \mathrm{ev}_{2}\right)\left(X_{0,2, k A_{1}}\right)$ lies in $D \times_{Z} D$, which 
has dimension $n+r-2$. The pushforward of the virtual fundamental class, $\left(\mathrm{ev}_{1} \times \mathrm{ev}_{2}\right)_{*}\left[X_{0,2, k A_{1}}\right]^{\mathrm{vir}}$, is supported on this set. On the other hand, the virtual dimension of $X_{0,2, k A_{1}}$ is $n-1+k(r-1)$; in particular, for $k \geq 2$ the virtual dimension is at least $n+2 r-3$. Since the virtual dimension is greater than the dimension of the set on which the pushforward is supported, $\left(\mathrm{ev}_{1} \times\right.$ $\left.\mathrm{ev}_{2}\right)_{*}\left[X_{0,2, k A_{1}}\right]^{\mathrm{vir}}$ vanishes and so too does the Gromov-Witten invariant.

We now prove that the invariants $\left\langle\alpha_{1}, \alpha_{2}\right\rangle_{0,2, A_{1}}^{X}$ can be calculated as claimed. The class $A_{1}$ is irreducible and reduced and can only be represented as a line in the fiber of the exceptional locus, hence the moduli space of holomorphic maps $\mathbb{P}^{1} \rightarrow X$ with target class $A_{1}$ is compact and of expected dimension. In particular, the moduli space $X_{0,0, A_{1}}$ is given by the Grassmann bundle of lines in the fiber of $\mathbb{P}\left(N_{Z / Y}\right)$. It is smooth and of the expected dimension $n+r-4$. The Gromov-Witten invariants are therefore enumerative and can be calculated by counting curves of class $A_{1}$ on which the insertion classes are supported.

This is identical to the problem of counting lines in fibers over the exceptional locus on which $\iota^{*} \alpha_{1}$ and $\iota^{*} \alpha_{2}$ are supported. As the exceptional divisor is the blowup of the normal bundle, we are in the cases of Lemma 1 and are counting common fibers of $\Xi$ in the support of $\iota^{*} \alpha_{1}$ and $\iota^{*} \alpha_{2}$. This counting is carried out by pushdown via $\Xi$ and intersection in the cohomology of the base. This yields the claimed formula.

In the above discussion we have proved the following corollary to Theorem 1 .

Corollary 1. Let $X=\mathbb{P}(E)$ with $E \rightarrow \mathbb{P}^{n}$ be a Fano bundle satisfying Assumptions 1 and 2 and with the second extremal contraction $\Xi: \mathbb{P}(E) \rightarrow Y$ given by either

(1) the projectivization of a bundle $E^{\prime} \rightarrow Y$,

(2) the blowup of a smooth subvariety $Z$ inside smooth $Y$;

then the quantum cohomology of $X$ can be reconstructed from the GromovWitten invariants of the form $\langle\alpha, \beta\rangle_{0,2, A_{1}}$ and $\langle\alpha, \beta\rangle_{0,2, A_{2}}$. These GromovWitten invariants are determined by Lemmas 1 and 6.

\section{§4. The geometry of rank 2 Fano 3-fold No. 17}

Our aim is to compute the quantum period sequence of $M$, the rank 2 Fano 3-fold No. 17 in Mori and Mukai [23]. The 3-fold $M$ can be embedded 
as a complete intersection in $X=\mathbb{P}(E)$ for some Fano bundle $E$, which we now describe.

Fix a vector space $V \simeq \mathbb{C}^{5}$ with $\mathbb{P}^{4}:=\mathbb{P}(V)$. Let $E:=\Omega_{\mathbb{P}(V)}^{2}(2)$ be the second wedge of the bundle of holomorphic differentials on $\mathbb{P}^{4}$, twisted by $\mathcal{O}(2)$. Let $X$ be the total space $\mathbb{P}(E)$. We can naturally view $X$ as the blowup of the Plücker embedding of the Grassmannian $G\left(2, V^{\star}\right) \subset \mathbb{P}\left(\bigwedge^{2} V^{\star}\right)$.

As above, let $P$ be the canonical map induced by $X$ 's bundle structure, and let $\Xi$ be the contraction induced by the second extremal ray. We illustrate this in the following diagram:

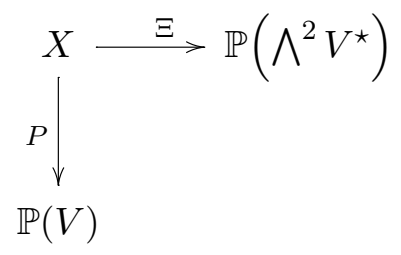

\subsection{Cohomology and extremal rays of $X$}

The ordinary cohomology of $X$ is determined by the Chern classes of $E$, which are easily obtained from the following exact sequence of bundles, taking the second wedge of the Euler sequence on $\mathbb{P}(V)$. By abuse of notation, we refer by $V^{\star} \otimes \mathcal{O}$ to the trivial $V^{\star}$ bundle on $\mathbb{P}(V)$ :

$$
0 \rightarrow E \rightarrow \bigwedge^{2} V^{\star} \otimes \mathcal{O} \stackrel{v\lrcorner}{\rightarrow} V^{\star} \otimes \mathcal{O}(1) \rightarrow \mathcal{O}(2) \rightarrow 0 .
$$

Here $v\lrcorner$ denotes the contraction by the vector $v \in V$ representing the point in $\mathbb{P}(V)$. From (2) it is clear that $X$ embeds into $\mathbb{P}(V) \times \mathbb{P}\left(\bigwedge^{2} V^{\star}\right) \cong$ $\mathbb{P}^{4} \times \mathbb{P}^{9}$. The total Chern class is $c(E)=1-3 p+5 p^{2}-5 p^{3}$ and hence

$$
H^{\bullet}(X)=\frac{\mathbb{C}[p, \xi]}{\left(p^{5}, \xi^{6}-3 p \xi^{5}+5 p^{2} \xi^{4}-5 p^{3} \xi^{3}\right)} .
$$

We check that $\xi$ is nef and prove that $E$ is a Fano bundle.

LEMMA 7. It holds that $\xi$ is nef.

Proof. Since $\xi$ is the relative hyperplane on $\mathbb{P}(E)$, it is a quotient of $P^{*} E^{\star}$. We have that $E^{\star}$ is generated by global sections (one sees this by dualizing (2)) so $\xi$ is also generated by global sections and is therefore nef. 
Since $-K_{X}=6 \xi+2 p$, and both $p$ and $\xi$ are nef, we see that $-K_{X}$ is ample; $X$ is Fano.

In $H_{2}(X, \mathbb{Z})$ we fix classes $A_{1}, A_{2}$. Recall that $A_{2}$ is the class of a line in the fiber $P: X \rightarrow \mathbb{P}(V)$. In this example, $A_{1}$ is the class of a line in an exceptional fiber of $\Xi: X \rightarrow \mathbb{P}\left(\bigwedge^{2} V^{\star}\right)$ and is isomorphic, by $P$, to a generic line in $\mathbb{P}^{n}$. We have

$$
p \cdot A_{1}=0, \quad \xi \cdot A_{1}=1, \quad p \cdot A_{2}=1, \quad \xi \cdot A_{2}=0
$$

from which follow $A_{1}=\operatorname{PD}\left(p^{3} \xi^{5}-3 p^{4} \xi^{4}\right)$ and $A_{2}=\operatorname{PD}\left(p^{4} \xi^{4}\right)$.

Lemma 8. The classes $A_{1}, A_{2}$ generate the extremal rays of the Mori cone.

Proof. Both $p$ and $\xi$ are nef, so, given the homology class $a A_{1}+b A_{2}$ of any curve $\mathrm{T}$, we have $a=\xi \cdot T \geq 0$ and $b=p \cdot T \geq 0$. On the other hand, both $A_{1}$ and $A_{2}$ are contained in the Mori cone and therefore form the extremal rays.

The following discussion shows that $X$ is given by the blowup of $\mathbb{P}\left(\bigwedge^{2} V^{\star}\right)$ along the Plücker embedding of $G\left(2, V^{\star}\right)$.

A point $[w] \in \mathbb{P}\left(\bigwedge^{2} V^{\star}\right)$ is represented by a 2 -form $w$. The fiber $\Xi^{-1}([w])$ over this point consists of the subset of points of $\mathbb{P}(V)$ which are represented by a vector $v$ annihilated by $w$. Considering $w$ as an antisymmetric $5 \times 5$ matrix $A: V \rightarrow V^{\star}$, it is clear that $w$ is generically of rank 4 , that is, there is a 1-dimensional space of vectors $v$ annihilated by $w$. So, generically, the fiber $\Xi^{-1}([w])$ is a point. When $w$ drops ranks it must be rank 2 , since we throw away the 0-form upon projectivization. Where $w$ is of rank 2, the fiber is a $\mathbb{P}^{2}$. The locus of rank-2 forms corresponds to those elements of $\bigwedge^{2} V^{\star}$ which are decomposable to $w_{1} \wedge w_{2}$, where $w_{1}, w_{2}$ are 1 -forms. This is exactly the Plücker embedding of $G\left(2, V^{\star}\right) \hookrightarrow \mathbb{P}\left(\bigwedge^{2} V^{\star}\right)$ as a codimension 3 subvariety. Hence $X$ is isomorphic to the blowup of $G\left(2, V^{\star}\right) \subset \mathbb{P}\left(\bigwedge^{2} V^{\star}\right)$.

Lemma 9. The rational map $\mathbb{P}\left(\bigwedge^{2} V^{\star}\right) \rightarrow \mathbb{P}(V)$ is given by the linear system of quadrics containing $G\left(2, V^{\star}\right)$.

Proof. The map sends a 2 -form $w$, thought of as an antisymmetric $5 \times 5$ matrix $A: V \rightarrow V^{\star}$, to its kernel. By a version of the Cramer rule, we can describe the map explicitly by sending $A$ to the vector of $4 \times 4$ Pfaffians:

$$
\operatorname{pf}(A)=\left(\operatorname{pf}_{0}(A), \ldots, \operatorname{pf}_{4}(A)\right) .
$$


The statement then reduces to the fact that these Pfaffians generate the ideal of $G(2, V) \subset \mathbb{P}\left(\bigwedge^{2} V^{\star}\right)$.

\section{2. $M$ as a complete intersection in $\mathbb{P}(E)$}

We denote by $M$ the Fano 3-fold No. 17 in the Mori-Mukai list of rank 2 Fano 3-folds (see [23]). According to Mori and Mukai, $M$ is the blowup of a 3-dimensional quadric $Q \subset \mathbb{P}^{4}$ with center $\Gamma \subset Q$, a nonsingular curve of genus 1 and degree 5 .

LEMMA 10. We have that $M$ is a complete intersection of type $p \cap \xi^{5}$ in $\mathbb{P}(E)$.

Proof. It is well known that the Plücker embedding $G\left(2, V^{\star}\right) \hookrightarrow \mathbb{P}\left(\bigwedge^{2} V^{\star}\right)$ is of degree 5 . Using adjunction, one can easily check that the curve given by the complete intersection of five general hyperplanes with the Grassmannian has trivial canonical bundle and hence is genus 1 ,

$$
\Gamma=h_{1} \cap \cdots \cap h_{5} \cap G\left(2, V^{\star}\right) \subset h_{1} \cap \cdots \cap h_{5} \cong \mathbb{P}^{4}
$$

all of this taking place in the natural ambient $\mathbb{P}\left(\bigwedge^{2} V^{\star}\right)$. Since $\Xi: X \rightarrow$ $\mathbb{P}\left(\bigwedge^{2} V^{\star}\right)$ is the blowup of $\mathbb{P}\left(\bigwedge^{2} V^{\star}\right)$ along the Plücker embedding of $G\left(2, V^{\star}\right)$, the discussion makes it clear that $M$ is the complete intersection

$$
M=\Xi^{*}\left(h_{1}\right) \cap \cdots \Xi^{*}\left(h_{5}\right) \cap \widetilde{Q} \subset X,
$$

where $\widetilde{Q}$ is the proper transform of a quadric containing $G\left(2, V^{\star}\right)$, that is, by Lemma 9 , a section of $p$.

Corollary 2. Let $-K_{M}=\left.(p+\xi)\right|_{X}$.

\section{$\S 5$. The quantum cohomology of $X$ and $M$}

In this section we use the reconstruction theorem (Theorem 1) to calculate the quantum cohomology of $X$. By passing to a certain generating function - the $J$-function - of $X$, we use quantum Lefschetz (see [10]) to obtain information about the quantum cohomology of $M$.

Since $X=\mathbb{P}(E)$ is the projectivization of a Fano bundle and the extremal contraction $\Xi$ is the blowup of $G(2,5) \subset \mathbb{P}^{9}$, the quantum cohomology follows from Corollary 1. 
We make use of the Schubert calculus for $G(2,5)$, following notational conventions from [16]. In particular, let $\sigma_{i}$ be the $i$ th Chern class of the tautological quotient bundle $Q$ on $G\left(2, V^{\star}\right)$. For example, $\sigma_{1}$ is the class of the Schubert cell of lines which intersect a given plane.

Let $N$ be the normal bundle to the embedding of $G\left(2, V^{\star}\right)$ in $\mathbb{P}\left(\bigwedge^{2} V^{\star}\right)$. The exceptional divisor $D$ is given by the projectivization of $N$. The normal bundle to the embedding into $\mathbb{P}\left(\bigwedge^{2} V^{\star}\right)$ is given by $Q^{\star}\left(2 \sigma_{1}\right)$, though for the sake of convenient relations in cohomology we will work instead with $Q^{\star}$ (of course $\mathbb{P}(N)$ and $\mathbb{P}\left(Q^{\star}\right)$ are isomorphic). The cohomology of this bundle is given by

$$
H^{\bullet}\left(\mathbb{P}\left(Q^{\star}\right)\right)=\frac{H^{\bullet}\left(G\left(2, V^{\star}\right)\right)[\eta]}{\left(\eta^{3}-\sigma_{1} \eta^{2}+\sigma_{2} \eta-\sigma_{3}\right)},
$$

where $\eta$ is the relative hyperplane class of $\mathbb{P}\left(Q^{\star}\right)$.

The following diagram describes the geometry of the situation:

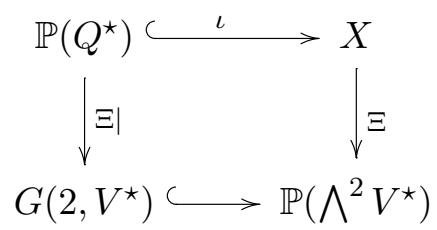

For the purpose of calculation, note that $\iota^{*} \xi=\sigma_{1}$ and $\iota^{*} p=\eta$.

THEOREM 2. We have

$$
Q H^{\bullet}(X)=\frac{\mathbb{C}\left[p, \xi, q_{1}, q_{2}\right]}{\left(R_{1}, R_{2}\right)}
$$

where

$$
R_{1}=p^{\star 5}-q_{1}^{2} p+2 q_{1}^{2} \xi+2 q_{1} p^{\star 3}-2 q_{1} p^{\star 2} \star \xi-q_{1} p \star \xi^{\star 2}-q_{1} \xi^{\star 3}
$$

and where

$$
R_{2}=\xi^{\star 6}-3 p \star \xi^{\star 5}+5 p^{\star 2} \star \xi^{\star 4}-5 p^{\star 3} \star \xi^{\star 3}-q_{2}-5 q_{1} p \star \xi^{\star 3}+10 q_{1} \xi^{\star 4}
$$

Proof. Quantum multiplication is determined by Corollary 1. From [26, Theorem 2.2], all that then remains is to evaluate the relations of the clas- 
sical cohomology (3), replacing classical multiplication with quantum multiplication, from which the statement follows.

\subsection{Quantum differential structure}

Our ultimate goal is to compute the $J$-function (see [13]), a generating function for certain genus 0 Gromov-Witten invariants, of $M$. As $M$ is a complete intersection in $X$, we will use the quantum Lefschetz theorem (see [10, Theorem 2, Corollary 6]), which expresses certain genus 0 GromovWitten invariants of $M$ in terms of invariants of the ambient space $X$. The input that we need for the quantum Lefschetz theorem is the $J$-function of $X$ as well as the direct sum of line bundles which describe $M$ as a complete intersection. In this section we describe a method to obtain an arbitrary number of terms in the power series expansion of the $J$-function of $X$, by solving a system of differential equations, called the quantum differential equations for $X$. Here we follow closely the excellent articles [17] and [18] by Guest.

Recall from Section 2 that $\phi_{1}, \ldots, \phi_{30}$ is the lexicographical basis of $H^{\bullet}(X ; \mathbb{Z})$ in $p$ and $\xi$, with $\phi^{1}, \ldots, \phi^{30}$ the dual basis given by the intersection pairing. The $J$-function of $X$ is the function $H^{2}(X ; \mathbb{C}) \rightarrow H^{\bullet}(X ; \mathbb{C}) \otimes$ $\mathbb{C}[[1 / z]]$ defined by

$$
J_{X}(t)=e^{t / z}\left(1+\sum_{\epsilon=1}^{30} \sum_{a, b \geq 0} e^{d \cdot t}\left\langle\frac{\phi^{\epsilon}}{z(z-\psi)}\right\rangle_{0,1, a A_{1}+b A_{2}}^{X}\right),
$$

where we expand $\left\langle\frac{\phi^{\epsilon}}{z(z-\psi)}\right\rangle_{0,1, a A_{1}+b A_{2}}$ as $\sum_{k \geq 0}\left\langle\phi^{\epsilon} \psi^{k}\right\rangle_{0,1, a A_{1}+b A_{2}} \frac{1}{z^{k+2}}$.

Since $t \in H^{2}(X, \mathbb{C})$ is nilpotent as an element of $H^{\bullet}(X)$, the expression $e^{t / z}$ makes sense in $H^{\bullet}(X) \otimes \mathbb{C}[[1 / z]]$.

By writing $t \in H^{2}(X ; \mathbb{C})$ as $t=t_{0}+t_{1} p+t_{2} \xi$, we regard the $J$-function as a function of $q_{1}=e^{t_{1}}, q_{2}=e^{t_{2}}$ :

$$
J_{X}(q)=q_{1}^{p / z} q_{2}^{\xi / z}\left(1+\sum_{\epsilon=1}^{30} \sum_{a, b \geq 0} q_{1}^{a} q_{2}^{b}\left\langle\frac{\phi^{\epsilon}}{z(z-\psi)}\right\rangle_{0,1, a A_{1}+b A_{2}}\right) .
$$

Here $q_{1}^{p / z}=\exp \left(p \log q_{1} / z\right)=\exp (t p / z)$.

The function $J_{X}$ satisfies a system of differential operators called quantum differential operators (see [18]). Let $M_{p}(q), M_{\xi}(q)$ denote the matrices of quantum multiplication by $p$ and $\xi$ with respect to the basis $\phi_{1}, \ldots, \phi_{30}$, which are easily obtained from Corollary 1 and the discussion above. The reader may find $M_{p}(q), M_{\xi}(q)$ in the Appendix. 
Consider the system of differential equations

$$
\begin{aligned}
& z q_{1} \frac{\partial}{\partial q_{1}} s=M_{p}(q) s, \\
& z q_{2} \frac{\partial}{\partial q_{2}} s=M_{\xi}(q) s,
\end{aligned}
$$

where $s$ is a vector-valued function of $t \in H^{2}(X ; \mathbb{C})$ (or equivalently, a multivalued vector function of $\left.q_{1}, q_{2}\right)$.

This system admits a fundamental solution matrix, the rows of which are given by vectors $J_{i}$ :

$$
S=\left(\begin{array}{ccccc}
- & - & J_{1} & - & - \\
- & - & J_{2} & - & - \\
& & \vdots & & \\
- & - & J_{30} & - & -
\end{array}\right) .
$$

The row vector $J_{30}$ is the expansion of the $J$-function as a vector-valued function in $H^{\bullet}(X ; \mathbb{C}) \otimes \mathbb{C}[[1 / z]]$ in terms of the basis $\phi_{1}, \ldots, \phi_{30}$. The differential system is equivalent to sixty differential equations in $J_{1}$ through $J_{30}$. By solving for the rows $J_{1}$ through $J_{29}$ in terms of $J_{30}$, we are left with thirty-one differential equations in $J_{X}$. By applying Gröbner basis techniques, we find a generating set for the ideal formed by these equations in the Weyl algebra. These (nonunique) differential equations are quantum differential equations and define $J_{X}$ up to scalar. Using the Rosenfeld-Gröbner tool in the differential algebra package of Maple 16, we obtain the following result.

LEMma 11. The ideal of quantum differential operators for $X$ is generated by

$$
\begin{aligned}
\Delta_{1}= & D_{2}^{10}-q_{2} D_{1}^{4}-2 q_{2} D_{1}^{3} D_{2}-4 q_{2} D_{1}^{2} D_{2}^{2}-3 q_{2} D_{1} D_{2}^{3}-q_{2} D_{2}^{4} \\
& -2 q_{1} q_{2} D_{1}^{2}-2 z q_{2} D_{1}^{3}-2 z q_{1} q_{2} D_{1} D_{2}-8 z q_{2} D_{1}^{2} D_{2}-3 q_{1} q_{2} D_{2}^{2} \\
& -9 z q_{2} D_{1} D_{2}^{2}-4 z q_{2} D_{2}^{3}-q_{1}^{2} q_{2}-4 z q_{1} q_{2} D_{1}-4 z^{2} q_{2} D_{1}^{2}-7 z q_{1} q_{2} D_{2} \\
& -9 z^{2} q_{2} D_{1} D_{2}-6 z^{2} q_{2} D_{2}^{2}-5 z^{2} q_{1} q_{2}-3 z^{3} q_{2} D_{1}-4 z^{3} q_{2} D_{2}-z^{4} q_{2}, \\
\Delta_{2}= & D_{1} D_{2}^{7}-2 D_{2}^{8}+5 q_{2} D_{1}^{2}+5 q_{2} D_{1} D_{2}+2 q_{2} D_{2}^{2}+5 q_{1} q_{2}+5 z q_{2} D_{1} \\
& +4 z q_{2} D_{2}+2 z^{2} q_{2},
\end{aligned}
$$


(6) $\Delta_{3}=5 D_{1}^{3} D_{2}^{3}-5 D_{1}^{2} D_{2}^{4}+3 D_{1} D_{2}^{5}-D_{2}^{6}+5 q_{1} D_{1} D_{2}^{3}-10 q_{1} D_{2}^{4}+q_{2}$,

$$
\begin{aligned}
\Delta_{4}= & D_{1}^{5}+2 q_{1} D_{1}^{3}-2 q_{1} D_{1}^{2} D_{2}-q_{1} D_{1} D_{2}^{2}-q_{1} D_{2}^{3}+q_{1}^{2} D_{1}+2 z q_{1} D_{1}^{2} \\
& -2 q_{1}^{2} D_{2}-3 z q_{1} D_{1} D_{2}-2 z q_{1} D_{2}^{2}+z^{2} q_{1} D_{1}-2 z^{2} q_{1} D_{2},
\end{aligned}
$$

where $D_{i}=z q_{i} \frac{\partial}{\partial q_{i}}$.

Corollary 3. We have that $J$ satisfies $\Delta_{1} J=\Delta_{2} J=\Delta_{3} J=\Delta_{4} J=0$.

The identity component of $J$, denoted $J^{0}$, is a power series in $q_{1}$ and $q_{2}$, that is, $J^{0}=\left(J, \phi^{0}\right)=\sum_{i, j \geq 0} c_{i, j} q_{1}^{i} q_{2}^{j}$. The differential system gives recursion relations for the coefficients in this power series. The coefficients are fixed by demanding that $c_{0,0}=1$. We can find $J^{0}$ up to arbitrary order, and by observation of finite terms try to find a general solution for the coefficients $c_{i, j}$ which solves the differential system. Such a solution, however, has not been forthcoming. We present the coefficients $c_{i, j}$ for $i \leq 7, j \leq 7$ in the following matrix $A=\left(a_{i, j}\right)$. We have cleared the denominators by setting $a_{i+1, j+1}=i !^{2} j !^{6} c_{i, j}$ :

$$
A:=\left[\begin{array}{cccccccc}
1 & 1 & 1 & 1 & 1 & 1 & 1 & 1 \\
0 & 5 & 20 & 51 & 104 & 185 & 300 & 455 \\
0 & 4 & 73 & 447 & 1756 & 5320 & 13539 & 30373 \\
0 & 0 & 90 & 1445 & 10904 & 55220 & 216110 & 703955 \\
0 & 0 & 36 & 2148 & 33001 & 282085 & 1690515 & 7926751 \\
0 & 0 & 0 & 1500 & 54500 & 819005 & 7606080 & 51405305 \\
0 & 0 & 0 & 400 & 50350 & 1447150 & 21460825 & 211463875 \\
0 & 0 & 0 & 0 & 24500 & 1590050 & 39750270 & 584307365
\end{array}\right] .
$$

We remark that the coefficients are all zero below the leading slant diagonal. Additionally, in the form presented where we have cleared denominators, the leading diagonal $a_{i, i}$ is given by the Apéry numbers (see [24]), so we expect that a closed formula may be hard to find. The occurrence of the Apéry numbers here may indicate hidden modular symmetries of $J_{0}$ (see [5], [27]). Golyshev has observed a striking connection between the quantum differential equations for Fano 3-folds of Picard rank 1 and modular forms (see [15]), and it is possible that this connection persists to the case of higher Picard rank.

\subsection{The regularized quantum period sequence for $M$}

As we noted in the preceding section, given a complete intersection $M \subset$ $X$, quantum Lefschetz (see [10]) allows us to calculate part of the $J_{M}$ from 
$J_{X}$ and the Chern classes of the line bundles defining the intersection. In our example, we are only interested in the identity component of the $J$-function; quantum Lefschetz provides the entirety of $J_{M}^{0}$ from $J_{X}^{0}$. Our aim is not to explain how quantum Lefschetz works, but how one uses it in practical examples.

With this in mind, we briefly outline the process of applying quantum Lefschetz. The following statements hold for any ambient space $X$ and complete intersection $M$.

Let $\mathcal{E}=\bigoplus L_{i}$ be the direct sum of line bundles corresponding to $M$, and let $\rho_{i}=c_{1}\left(L_{i}\right)$ be the first Chern class of the line bundle summands. Given the $J$-function of $X, J_{X}(t, z)=\sum_{d \in H_{2}(X)} J_{d}(t, z) q^{d}$, one forms the hypergeometric modification

$$
I_{X, M}(t, z)=\sum_{d} J_{d}(t, z) q^{d} \prod_{i} \prod_{k=1}^{\rho_{i} \cdot d}\left(\rho_{i}+k z\right)
$$

We also consider a formal function (with same domain and target) $J_{X, M}(t$, $z)$, defined in [10], which has the property

$$
e(\mathcal{E}) J_{X, M}\left(j^{*} u, z\right)=j_{\star} J_{M}(u, z),
$$

where $e(\mathcal{E})=\prod_{i} \rho_{i}$ is the Euler class of the bundle $\mathcal{E}$. The relation between $J_{M}$ and $J_{X}$ is indirectly realized by the mirror map, which relates $I_{X, M}$ to $J_{X, M}$. The mirror map is determined by comparing the asymptotics of the expressions. Considered as a power series in $z^{-1}, J_{X, M}$ is the unique function with the form $J_{X, M}=z+t+O\left(z^{-1}\right)$. We may write $I_{X, M}$ in the form $F(t) z+G^{0}(q, t) \phi_{0}+\sum_{i=1}^{r} G^{i}(t) \phi_{i}+O\left(z^{-1}\right)$. By homogeneity considerations writing $I_{X, M}$ in this form is practicable, even in the case that $J_{X}$ is only known up to finite order in $q$, as in the case in point. The mirror map is given by

$$
\frac{I_{M, X}}{F(t)}=J_{X, M}\left(\frac{G^{0}(q, t)}{F(t)} \phi_{0}+\sum_{i=1}^{r} \frac{G^{i}(t)}{F(t)} \phi_{i}, z\right)=e^{\frac{G^{0}(q, t)}{F(t)} \phi_{0}} J_{X, M}(\tau, q),
$$

where $\tau=\sum_{i=1}^{r} \frac{G^{i}(t)}{F(t)} \phi_{i}$. The second equality follows from the string equation and the definition of $J$.

The procedure may be summarized as follows.

(1) Calculate $J_{X}$ 
(2) Produce the hypergeometric modification $I_{X, M}$.

(3) Calculate the mirror map from the asymptotics of $I_{X, M}$.

(4) Produce $J_{X, M}$ from $I_{X, M}$ using the mirror map.

Note that we do not obtain the entirety of $J_{M}$ by comparison with $J_{X, M}$ : some information is lost in the pushforward. However, we can recover the full identity component $J_{M}^{0}$ by following the described method with $J_{X}^{0}$, since pushforward of the identity is cup with the Euler class.

We now return to our particular example and previous definitions for $M \subset X=\mathbb{P}\left(\Omega^{2}(2)\right)$ and, proceeding as above, produce $J_{X}$. As described in Section 4.2, $M$ is a complete intersection in $X$ given by the intersection of one hyperplane of class $\operatorname{PD}(p)$ by 5 of $\operatorname{PD}(\xi)$. The hypergeometric modification of $J_{X}=\sum_{a, b} J_{a, b}(t, z) q_{1}^{a} q_{2}^{b}$ is given by

$$
I_{X, M}(t, z)=\sum_{a, b} J_{a, b}(t, z) q_{1}^{a} q_{2}^{b} \prod_{k=1}^{a}(p+k z) \prod_{k=1}^{b}(\xi+k z)^{5} .
$$

Restricting our attention to the identity component $J_{X}^{0}=\sum_{i, j} c_{i, j} q_{1}^{i} q_{2}^{j}$, we have $I_{X, M}^{0}=\left(I_{X, M}, \phi^{0}\right)=\sum_{i, j} d_{i, j} q_{1}^{i} q_{2}^{j}$, with $d_{i, j}=c_{i, j} i !(j !)^{5}$.

If we set the degree of $\operatorname{deg}(z)=1$ and $\operatorname{deg}\left(t^{i}\right)=1-\operatorname{deg}\left(\phi_{i}\right), J_{X}$ is known to be homogeneous of degree 1 . One can see that in our example the only possible contributions to the mirror map come from the identity component, $J_{X}^{0}$, and in particular we need only consider $c_{0,0}, c_{1,0}, c_{0,1}$. We find that $F(t)=1, G^{0}(q, t)=1+q_{2}, G^{1}(t)=G^{2}(t)=1$.

LEMMA 12. The mirror map is given by

$$
J_{M, X}\left(\tau_{0}, t_{1}, t_{2}, z\right)=I_{M, X}\left(t_{0}, t_{1}, t_{2}, z\right),
$$

where $\tau_{0}=t_{0}+q_{1}$. We can more conveniently write this as

$$
J_{M, X}\left(t_{0}, t_{1}, t_{2}, z\right)=e^{-q_{1}} I_{M, X}\left(t_{0}, t_{1}, t_{2}, z\right) .
$$

To produce the quantum period sequence from $J_{M}^{0}$, we restrict $t$ to the anticanonical direction in $H^{2}(M ; \mathbb{C})$. As previously stated, $-K_{M}=p+\xi$, and so restricting to the anticanonical direction has the effect of setting $q_{1}=q_{2}=q=e^{t}$. The effect on $J_{M}^{0}$ is to collapse the sum to a power series in one variable with coefficients $d_{i}=\sum_{j+k=i} c_{j, k}$. The first ten terms in the period sequence are: 1, 0, 10, 42, 414, 3300, 29890, 275940, 2608270, 25305000 . 
Since it is known that $J_{M}^{0}$ satisfies quantum differential equations, the period sequence also does. Given sufficient entries in the power series of the period sequence, we may find the differential operator which annihilates the sequence.

The Picard-Fuchs operator for the regularized period sequence of $M$ is given by

(9)

$$
\begin{aligned}
- & -7727940 t^{9} D^{4}-47452732 t^{8} D^{4}-177279400 t^{9} D^{3}-51239477 t^{7} D^{4} \\
- & 400876912 t^{8} D^{3}-620477900 t^{9} D^{2}-28719434 t^{6} D^{4} \\
- & 363088702 t^{7} D^{3}-1218943172 t^{8} D^{2}-886397000 t^{9} D \\
- & 8782543 t^{5} D^{4}-169273876 t^{6} D^{3}-958664473 t^{7} D^{2} \\
- & 1562482112 t^{8} D-425470560 t^{9}-1322684 t^{4} D^{4} \\
- & 42555106 t^{5} D^{3}-384463114 t^{6} D^{2}-1102964660 t^{7} D \\
- & 696963120 t^{8}-37187 t^{3} D^{4}-5281118 t^{4} D^{3}-80112855 t^{5} D^{2} \\
- & 392394560 t^{6} D-456149412 t^{7}+13026 t^{2} D^{4}-238966 t^{3} D^{3} \\
- & 7132816 t^{4} D^{2}-69331328 t^{5} D-148485888 t^{6}+995 t D^{4} \\
- & 11442 t^{2} D^{3}-3879 t^{3} D^{2}-4318688 t^{4} D-22881836 t^{5} \\
- & 24 D^{4}-2278 t D^{3}+16030 t^{2} D^{2}+146332 t^{3} D-928456 t^{4} \\
+ & 24 D^{3}+35 t D^{2}+9600 t^{2} D+76072 t^{3}+1920 t^{2},
\end{aligned}
$$

where $D=t \frac{d}{d t}$.

This matches the Picard-Fuchs operator predicted by the mirror polytope in [9].

\section{Appendix. Quantum multiplication matrices}




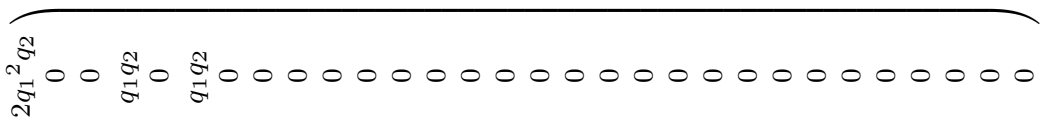

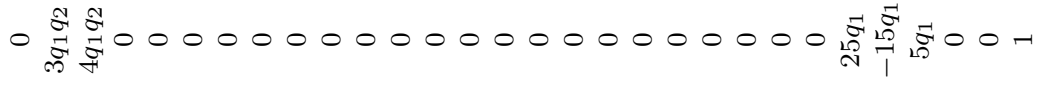

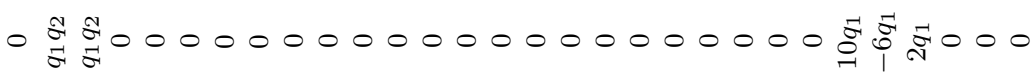

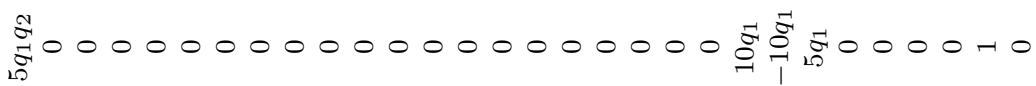

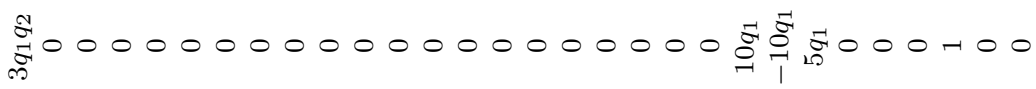

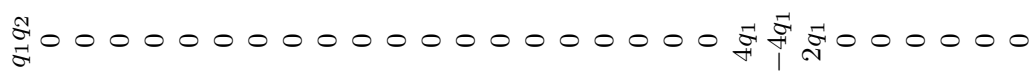
$00000000000000000000000000-000$ 000000000000000000 H H 000000000000000000 F 0000000000000000 F $00000000000000000000000-1000000$ $0000000000000000000000-10000000$ 0000000000000 F 0000000000000 F 00000000000 F F $F$ F $000000000000000000-100000000000$ $00000000000000000-1000000000000$ 00000000 F F 00000000 F 000000 F $F$ F 700000000000000000000 $0000000000000-10000000000000000$ $000000000000-00000000000000000$ 0000 F F 0000 i $70000-10000000000000000000$ $00000000-1000000000000000000000$ $0000000-10000000000000000000000$ 0 I $0000-0000000000000000000000000$ $000-00000000000000000000000000$ 


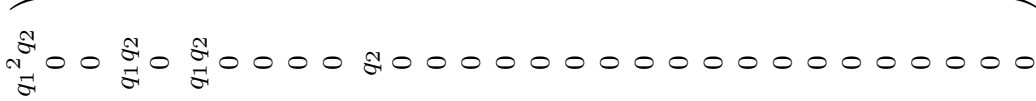
0 票栗

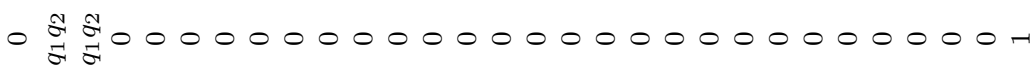
I $\mathbb{m}_{1}^{2} 000000000000000000000000000-10$ I

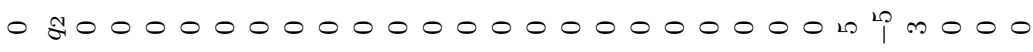
$00000000000000000000000000-1000$ $0000000000000000000000000-10000$ $000000000000000000000000-100000$ HO0 $00000000000000000000000-1000000$ $0000000000000000000000-0000000$ $000000000000000000000-100000000$ $00000000000000000000-000000000$ $0000000000000000000-0000000000$ $000000000000000000-100000000000$ $00000000000000000-1000000000000$ $0000000000000000-10000000000000$ $000000000000000-100000000000000$ $00000000000000-000000000000000$ $0000000000000-0000000000000000$ $000000000000-00000000000000000$ $00000000000-1000000000000000000$ $000000000-100000000000000000000$ $00000000-000000000000000000000$ $0000000-10000000000000000000000$ $00000-000000000000000000000000$ $0000-10000000000000000000000000$ $00-000000000000000000000000000$ 
Acknowledgments. This article is the outcome of work conducted for my Ph.D. dissertation at Imperial College London, with funding from the Engineering and Physical Sciences Research Council (EPSRC-United Kingdom). I would like to thank my supervisors Alessio Corti and Tom Coates for their advice and support. I would also like to thank János Kollár for pointing out examples of smooth projective varieties $M$, on which there exist classes $A \in H_{2}(M ; \mathbb{Z})$ where, for $m>1, m A$ is the class of an effective curve, while $A$ is not. I would also like to thank Martin Guest and Hiroshi Iritani for helpful comments during a recent trip to Japan. Finally, I would like to thank an anonymous referee for offering extremely valuable comments, suggestions and, in particular, simplifications to the proof of Lemma 6.

\section{REFERENCES}

[1] V. Ancona and M. Maggesi, "On the quantum cohomology of Fano bundles over projective spaces" in The Fano Conference (Turin, 2002), University of Turin, Turin, 2004, 81-98. MR 2112569.

[2] V. Ancona, T. Peternell, and J. A. Wiśniewski, Fano bundles and splitting theorems on projective spaces and quadrics, Pacific J. Math. 163 (1994), 17-42. MR 1256175.

[3] K. Behrend, Gromov-Witten invariants in algebraic geometry, Invent. Math. 127 (1997), 601-617. MR 1431140. DOI 10.1007/s002220050132.

[4] K. Behrend and B. Fantechi, The intrinsic normal cone, Invent. Math. 128 (1997), 45-88. MR 1437495. DOI 10.1007/s002220050136.

[5] F. Beukers, "Irrationality proofs using modular forms" in Journées arithmétiques de Besançon (Besançon, 1985), Astérisque 147-148, Soc. Math. France, Paris, 1987, 271-283, 345. MR 0891433.

[6] I. Ciocan-Fontanine, B. Kim, and C. Sabbah, The abelian/nonabelian correspondence and Frobenius manifolds, Invent. Math. 171 (2008), 301-343. MR 2367022. DOI 10.1007/s00222-007-0082-x.

[7] H. Clemens, J. Kollár, and S. Mori, Higher-dimensional Complex Geometry, Astérisque 166, Soc. Math. France, Paris, 1989. MR 1004926.

[8] T. Coates, A. Corti, S. Galkin, V. Golyshev, and A. Kasprzyk, Mirror symmetry and Fano manifolds, to appear in Proceedings of the 6th European Congress of Mathematics, preprint, arXiv:1212.1722v1 [math.AG].

[9] T. Coates, A. Corti, S. Galkin, V. Golyshev, and A. Kasprzyk, (Fano search website), http://www . fanosearch. net (accessed 9 September 2014).

[10] T. Coates and A. Givental, Quantum Riemann-Roch, Lefschetz and Serre, Ann. of Math. (2) 165 (2007), 15-53. MR 2276766. DOI 10.4007/annals.2007.165.15.

[11] W. Fulton, Intersection Theory, 2nd ed., Ergeb. Math. Grenzgeb. (3) 2, Springer, Berlin, 1998. MR 1644323. DOI 10.1007/978-1-4612-1700-8.

[12] W. Fulton and R. Pandharipande, "Notes on stable maps and quantum cohomology" in Algebraic Geometry (Santa Cruz, Calif., 1995), Proc. Sympos. Pure Math. 62 (Part 2), Amer. Math. Soc., Providence, 1997, 45-96. MR 1492534.

[13] A. B. Givental, Equivariant Gromov-Witten invariants, Int. Math. Res. Not. IMRN 13 (1996), 613-663. MR 1408320. DOI 10.1155/S1073792896000414. 
[14] - "A mirror theorem for toric complete intersections" in Topological Field Theory, Primitive Forms and Related Topics (Kyoto, 1996), Progr. Math. 160, Birkhäuser, Boston, 1998, 141-175. MR 1653024.

[15] V. V. Golyshev, "Classification problems and mirror duality" in Surveys in Geometry and Number Theory: Reports on Contemporary Russian Mathematics, London Math. Soc. Lecture Note Ser. 338, Cambridge University Press, Cambridge, 2007, 88-121. MR 2306141. DOI 10.1017/CBO9780511721472.004.

[16] P. Griffiths and J. Harris, Principles of Algebraic Geometry, reprint of 1978 original, Wiley Classics Lib., Wiley, New York, 1994. MR 1288523. DOI 10.1002/9781118032527.

[17] M. A. Guest, "Introduction to homological geometry, I" in Integrable Systems, Geometry, and Topology, AMS/IP Stud. Adv. Math. 36, Amer. Math. Soc., Providence, 2006, 83-121. MR 2222513.

[18] - "Introduction to homological geometry, II" in Integrable Systems, Geometry, and Topology, AMS/IP Stud. Adv. Math. 36, Amer. Math. Soc., Providence, 2006, 123-150. MR 2222514.

[19] J. Kock and I. Vainsencher, An Invitation to Quantum Cohomology: Kontsevich's Formula for Rational Plane Curves, Progr. Math. 249, Birkhäuser, Boston, 2007. MR 2262630.

[20] J. Kollár, Holomorphic and pseudo-holomorphic curves on rationally connected varieties, Port. Math. 67 (2010), 155-179. MR 2662865. DOI 10.4171/PM/1863.

[21] M. Kontsevich and Yu. Manin, Gromov-Witten classes, quantum cohomology, and enumerative geometry, Comm. Math. Phys. 164 (1994), 525-562. MR 1291244.

[22] J. Li and G. Tian, Virtual moduli cycles and Gromov-Witten invariants of algebraic varieties, J. Amer. Math. Soc. 11 (1998), 119-174. MR 1467172. DOI 10.1090/S0894-0347-98-00250-1.

[23] S. Mori and S. Mukai, "Extremal rays and Fano 3-folds" in The Fano Conference (Turin, 2002), University of Turin, Turin, 2004, 37-50. MR 2112566.

[24] - The On-Line Encyclopedia of Integer Sequences, Apéry numbers, http://oeis.org/A005259 (accessed 8 September 2014).

[25] Z. Qin and Y. Ruan, Quantum cohomology of projective bundles over $\mathbf{P}^{n}$, Trans. Amer. Math. Soc. 350, no. 9 (1998), 3615-3638. MR 1422617. DOI 10.1090/S0002-9947-98-01968-0.

[26] B. Siebert and G. Tian, On quantum cohomology rings of Fano manifolds and a formula of Vafa and Intriligator, Asian J. Math. 1 (1997), 679-695. MR 1621570.

[27] D. Zagier, "Integral solutions of Apéry-like recurrence equations" in Groups and Symmetries, CRM Proc. Lecture Notes 47, Amer. Math. Soc., Providence, 2009, 349-366. MR 2500571.

Department of Mathematics

Imperial College London

London, SW7 2AZ

United Kingdom

a.strangeway09@imperial.ac.uk 